PROCEEDINGS OF THE

AMERICAN MATHEMATICAL SOCIETY

Volume 126, Number 10, October 1998, Pages 2857-2861

S $0002-9939(98) 04736-4$

\title{
A NEW CHARACTERISTIC OF MÖBIUS TRANSFORMATIONS BY USE OF APOLLONIUS QUADRILATERALS
}

\author{
HIROSHI HARUKI AND THEMISTOCLES M. RASSIAS \\ (Communicated by Palle E. T. Jorgensen)
}

\begin{abstract}
The purpose of this paper is to give a new invariant characteristic property of Möbius transformations from the standpoint of conformal mapping. To this end a new concept of "Apollonius quadrilaterals" is used.
\end{abstract}

\section{INTRODUCTION AND STATEMENT OF RESULTS}

Throughout the paper, unless otherwise stated, let $w=f(z)$ be a nonconstant meromorphic function of a complex variable $z$ in $|z|<+\infty$. We consider the following Property A:

Property A. The function $w=f(z)$ transforms circles on the $z$-plane onto circles on the $w$-plane, including straight lines among circles.

The well-known principle of circle-transformation (see [1], [4], [5], [7, p. 160]) reads:

Theorem A. $w=f(z)$ satisfies Property A iff $w=f(z)$ is a Möbius transformation.

Before stating Property B we shall give the definition of Apollonius quadrilaterals on the complex plane.

Definition. Let $A B C D$ be an arbitrary quadrilateral (not necessarily simple) on the complex plane. If $\overline{A B} \cdot \overline{C D}=\overline{B C} \cdot \overline{D A}$ holds, then $A B C D$ is said to be an Apollonius quadrilateral. $\left(\overline{A B}=\left|z_{1}-z_{2}\right|\right.$ where $z_{1}, z_{2}$ are the complex numbers corresponding to the points $A, B$ respectively.)

Example 1. Each of a square, a rhombus and a kite is an Apollonius quadrilateral.

Example 2. If from a point outside a circle a secant and two tangents are drawn, then the four points on the circle form an Apollonius quadrilateral.

Proof. The proof follows from the following theorem (see [3, p. 7]): If a chord of a circle is drawn from the point of contact of a tangent, the angle made by the chord with the tangent is equal to the angle subtended by the chord at a point on that part of the circumference which lies on the far side of the chord.

Received by the editors February 18, 1997.

1991 Mathematics Subject Classification. Primary 32A20.

(C)1998 American Mathematical Society 
We may now state Property B. In section 3 we shall prove that Property A implies the following Property B.

Property B. Suppose that $w=f(z)$ is analytic and univalent in a non-empty domain $R$ on the $z$-plane. Let $A B C D$ be an arbitrary Apollonius quadrilateral contained in $R$. If we set $A^{\prime}=f(A), B^{\prime}=f(B), C^{\prime}=f(C), D^{\prime}=f(D)$, then $A^{\prime} B^{\prime} C^{\prime} D^{\prime}$ is also an Apollonius quadrilateral on the $w$-plane.

The purpose of this paper is to give a new invariant characteristic property of Möbius transformations from the standpoint of conformal mapping and to give a new proof of the only if part of Theorem A. The results in this paper are contained in the following theorem and its corollary.

Main Theorem. The function $w=f(z)$ satisfies Property $B$ iff $w=f(z)$ is a Möbius transformation.

Corollary. This theorem gives a new proof of the only if part of Theorem A.

\section{LEMMAS}

In sections 3 and 4 we shall apply the following three lemmas:

Lemma 1. If the function $w=f(z)$ satisfies Property $A$, then $w=f(z)$ is univalent in $|z|<+\infty$.

Proof. See [4].

Lemma 2 (The Theorem of Apollonius). We include straight lines among circles.

(i) The locus of a point, which moves so that the ratio of its distances from two fixed points is constant, is a circle with respect to which those points are inverse.

(ii) If $A, B$ are two fixed points inverse with respect to a given circle, then the ratio $\frac{\overline{P A}}{\overline{P B}}$ is constant for all positions of $P$ on that circle.

Proof. (i) See [6, p. 129].

(ii) See $[6$, pp. 129-130].

Lemma 3. If the function $w=f(z)$ is analytic and univalent in a non-empty domain $R$, then $f^{\prime}(z) \neq 0$ in $R$.

Proof. See [9, p. 302].

\section{Proof that Property A implies Property B}

Suppose that $w=f(z)$ is analytic in a non-empty domain $R$ on the $z$-plane. Since, by hypothesis, $w=f(z)$ satisfies Property A, by Lemma $1 w=f(z)$ is univalent in $|z|<+\infty$. Thus $w=f(z)$ is univalent in $R$. Let $A B C D$ be an arbitrary Apollonius quadrilateral contained in $R$. Then, by definition we obtain

$$
\overline{A B} \cdot \overline{C D}=\overline{B C} \cdot \overline{D A},
$$

and so

$$
\frac{\overline{A B}}{\overline{C B}}=\frac{\overline{A D}}{\overline{C D}} .
$$

We denote (1) by $N$. By Lemma 2 (i) the locus of a point, which moves so that the ratio of its distances from two fixed points $A, C$ is $N$ (= const), is a circle with 
respect to which $A, C$ are inverse. We denote the above circle of Apollonius by $K$ and let $K^{\prime}=f(K)$. Since, by hypothesis, $w=f(z)$ satisfies Property A, $K^{\prime}$ is also a circle on the $w$-plane. If we set $A^{\prime}=f(A), B^{\prime}=f(B), C^{\prime}=f(C), D^{\prime}=f(D)$, then by the fact that $B, D$ are on $K$ and by $K^{\prime}=f(K) B^{\prime}, D^{\prime}$ are on the circle $K^{\prime}$. Since $A, C$ are inverse with respect to the circle $K$, by the Reflection Principle [10, p. 155] of analytic functions it follows that $A^{\prime}=f(A), C^{\prime}=f(C)$ are also inverse with respect to the circle $K^{\prime}=f(K)$. Consequently, by Lemma 2 (ii) we obtain

$$
\overline{\overline{C^{\prime} B^{\prime}}}=\overline{\overline{A^{\prime} D^{\prime}}}
$$

which implies

$$
\overline{A^{\prime} B^{\prime}} \cdot \overline{C^{\prime} D^{\prime}}=\overline{B^{\prime} C^{\prime}} \cdot \overline{D^{\prime} A^{\prime}} .
$$

Therefore, the quadrilateral $A^{\prime} B^{\prime} C^{\prime} D^{\prime}$ on the $w$-plane is also an Apollonius quadrilateral, and so we get the desired result.

\section{Proof of the Main theorem}

If $w=f(z)$ is a Möbius transformation, then, by the if part of Theorem A, $w=f(z)$ satisfies Property A. Thus, by the result in section $3, w=f(z)$ satisfies Property B. Then, we shall prove the only if part of the main theorem, i.e., if $w=f(z)$ satisfies Property B, then $w=f(z)$ is a Möbius transformation. Since $w=f(z)$ is analytic and univalent in the domain $R$, by Lemma 3 it follows that

$$
f^{\prime}(z) \neq 0 .
$$

If $x$ is an arbitrarily fixed point of $R$, then, by (2) we obtain

$$
f^{\prime}(x) \neq 0 .
$$

Let $E$ be the point represented by $x$. Since $E \in R$, there exists a positive real number $r$ such that the $r$ closed neighbourhood of $E$ is contained in $R$. We denote this closed neighbourhood by $V$. Throughout the rest of the proof let $A B C D$ denote an arbitrary square which is contained in $V$ and whose centre is at $E$. Here the sense of $A, B, C, D$ is counterclockwise. Since $A B C D$ is a square contained in $V$, we can represent $A, B, C, D$ by complex numbers

$$
x+y, x+i y, x-y, x-i y \quad(|y| \leq r),
$$

respectively. Since $w=f(z)$ is univalent in $R, A^{\prime}(=f(A)), B^{\prime}(=f(B)), C^{\prime} \quad(=$ $f(C)), D^{\prime}(=f(D))$ are different points. Since, by hypothesis, $A^{\prime} B^{\prime} C^{\prime} D^{\prime}$ is an Apollonius quadrilateral, by definition we obtain

$$
\overline{A^{\prime} B^{\prime}} \cdot \overline{C^{\prime} D^{\prime}}=\overline{B^{\prime} C^{\prime}} \cdot \overline{D^{\prime} A^{\prime}} .
$$

Since $A^{\prime}, B^{\prime}, C^{\prime}, D^{\prime}$ are represented by

$$
f(x+y), f(x+i y), f(x-y), f(x-i y),
$$

respectively, we obtain

$$
\begin{aligned}
& \overline{A^{\prime} B^{\prime}}=|f(x+y)-f(x+i y)|, \\
& \overline{B^{\prime} C^{\prime}}=|f(x+i y)-f(x-y)|, \\
& \overline{C^{\prime} D^{\prime}}=|f(x-y)-f(x-i y)|,
\end{aligned}
$$




$$
\overline{D^{\prime} A^{\prime}}=|f(x-i y)-f(x+y)| .
$$

Substituting (5), (6), (7), (8) into (4) yields

$$
\begin{aligned}
& |(f(x+y)-f(x+i y))(f(x-y)-f(x-i y))| \\
& \quad=|(f(x+i y)-f(x-y))(f(x-i y)-f(x+y))|
\end{aligned}
$$

and therefore

$$
\frac{|(f(x+y)-f(x+i y))(f(x-y)-f(x-i y))|}{|(f(x+i y)-f(x-y))(f(x-i y)-f(x+y))|}=1 .
$$

If we set

$$
g(y)=\frac{(f(x+y)-f(x+i y))(f(x-y)-f(x-i y))}{(f(x+i y)-f(x-y))(f(x-i y)-f(x+y))},
$$

then, by (9) we have

$$
|g(y)|=1 .
$$

Since the numerator and the denominator of $g(y)$ in (10) are analytic for all $y$ satisfying $0<|y| \leq r$ and since, by the fact that $w=f(z)$ is univalent in $R$, the denominator of $g(y)$ in (10) never vanishes in $0<|y| \leq r, g(y)$ is analytic in $0<|y| \leq r$. Next we will prove that $g(y)$ is also analytic at $y=0$. As $y \rightarrow 0$, by L'Hopital's Rule (see [2]) and by (3) we have

$$
\frac{f(x+y)-f(x+i y)}{f(x+i y)-f(x-y)} \rightarrow \frac{f^{\prime}(x)-i f^{\prime}(x)}{i f^{\prime}(x)+f^{\prime}(x)}=\frac{1-i}{1+i}
$$

and

$$
\frac{f(x-y)-f(x-i y)}{f(x-i y)-f(x+y)} \rightarrow \frac{-f^{\prime}(x)+i f^{\prime}(x)}{-i f^{\prime}(x)-f^{\prime}(x)}=\frac{1-i}{1+i} .
$$

Hence, by (10), (12), (13), as $y \rightarrow 0$,

$$
g(y) \rightarrow\left(\frac{1-i}{1+i}\right)^{2}=-1 .
$$

If we define

$$
g(0)=-1
$$

by (14), by Riemann's Theorem on removable singularities, the function $g(y)$ is analytic at $y=0$. Furthermore, by (15) the equality (11) still holds at $y=0$. Thus $g(y)$ is analytic in $|y| \leq r$ and $|g(y)|=1$ holds in $|y| \leq r$. Therefore, by the Maximum Modulus Principle [8, p. 141] of analytic functions we obtain

$$
g(y)=K
$$

in $|y| \leq r$, where $K$ is a complex constant.

Setting $y=0$ in (16) and using (15) it follows

$$
K=-1 \text {. }
$$

By (10), (16), (17) we obtain

$$
\begin{aligned}
& (f(x+y)-f(x+i y))(f(x-y)-f(x-i y)) \\
& \quad+(f(x+i y)-f(x-y))(f(x-i y)-f(x+y))=0
\end{aligned}
$$

for all $y$ satisfying $|y| \leq r$. 
Using Leibnitz's Rule for differentiation, differentiating both sides of (18) four times with respect to $y$, setting $y=0$ and simplifying the resulting equality yields

$$
f^{\prime \prime \prime}(x) f^{\prime}(x)-\frac{3}{2} f^{\prime \prime}(x)^{2}=0 .
$$

Since $x \in R$ (the domain) was arbitrarily fixed, we can replace $x$ by a variable $z$ and thus by (19) we have

$$
f^{\prime \prime \prime}(z) f^{\prime}(z)-\frac{3}{2} f^{\prime \prime}(z)^{2}=0
$$

in $R$. By the Identity Theorem (see [7, p. 106]) the above equality holds in $|z|<$ $+\infty$. Hence

$$
\frac{f^{\prime \prime \prime}(z)}{f^{\prime}(z)}-\frac{3}{2}\left(\frac{f^{\prime \prime}(z)}{f^{\prime}(z)}\right)^{2}=0
$$

holds for all $z$ satisfying $f^{\prime}(z) \neq 0$.

Thus, the Schwarzian derivative of $f$ vanishes for all $z$ satisfying $f^{\prime}(z) \neq 0$. Therefore, by a well-known fact $f(z)$ is a Möbius transformation of $z$.

Proof of the Corollary. By hypothesis $w=f(z)$ satisfies Property A. Hence, by the result of section $3, w=f(z)$ satisfies Property B. Consequently, by the main theorem $w=f(z)$ is a Möbius transformation.

\section{REFERENCES}

1. J. Aczél and M. A. McKiernan, On the characterization of plane projective and complex Möbius transformation, Math. Nachr. 33 (1967), 315-337. MR 36:5806

2. J. Aczél, Functional equations and L'Hôpital's rule in an exact Poisson derivation, Amer. Math. Monthly 97 (1990), 423-426. CMP 9:11

3. H. S. M. Coxeter, Introduction to Geometry (5th ed.), John Wiley \& Sons, Inc., New YorkLondon-Sydney, 1966. MR 23:A1251

4. H. Haruki, A proof of the principle of circle-transformation by use of a theorem on univalent functions, L'Enseignement Mathématique 18 (1972), 145-146. MR 48:4312

5. H. Haruki and Th. M. Rassias, A new invariant characteristic property of Möbius transformations from the standpoint of conformal mapping, Journal of Mathematical Analysis and Applications 181 (1994), 320-327. MR 94m:30018

6. E. A. Maxwell, Geometry for Advanced Pupils, Oxford University Press, 1957.

7. Z. Nehari, Conformal Mapping, McGraw-Hill Book Co., New York, 1952. MR 13:640h

8. R. Nevanlinna and V. Paatero, Introduction to Complex Analysis, Addison-Wesley, New York, 1964. MR 39:415

9. L. L. Pennisi, L. I. Gordon and S. Lasher, Elements of Complex Variables, Holt, Rinehart and Winston, New York, 1963.

10. E. C. Titchmarsh, The Theory of Functions (2nd ed.), Clarendon Press, Oxford, 1939.

Department of Pure Mathematics, Faculty of Mathematics, University of Waterloo, Waterloo, Ontario, Canada N2L 3G1

Department of Mathematics, National Technical University of Athens, Zografou Campus, 15780 Athens, Greece

E-mail address: trassias@math.ntua.gr 